July 30, 2018

MITP/17-051 (Mainz)

\title{
Decay chain information on the newly discovered double charm baryon state $\Xi_{c c}^{++}$
}

\author{
Thomas Gutsche, ${ }^{1}$ Mikhail A. Ivanov, ${ }^{2}$ Jürgen G. Körner, ${ }^{3}$ and Valery E. Lyubovitskij ${ }^{1,4,5,6}$ \\ ${ }^{1}$ Institut für Theoretische Physik, Universität Tübingen, \\ Kepler Center for Astro and Particle Physics, Auf der Morgenstelle 14, D-72076 Tübingen, Germany \\ ${ }^{2}$ Bogoliubov Laboratory of Theoretical Physics, Joint Institute for Nuclear Research, 141980 Dubna, Russia \\ ${ }^{3}$ PRISMA Cluster of Excellence, Institut für Physik, \\ Johannes Gutenberg-Universität, D-55099 Mainz, Germany \\ ${ }^{4}$ Departamento de Física y Centro Científico Tecnológico de Valparaíso-CCTVal, \\ Universidad Técnica Federico Santa María, Casilla 110-V, Valparaíso, Chile \\ ${ }^{5}$ Department of Physics, Tomsk State University, 634050 Tomsk, Russia \\ ${ }^{6}$ Laboratory of Particle Physics, Tomsk Polytechnic University, 634050 Tomsk, Russia
}

\begin{abstract}
We interprete the new double charm baryon state found by the LHCb Collaboration in the invariant mass distribution of the set of final state particles $\left(\Lambda_{c}^{+} K^{-} \pi^{+} \pi^{+}\right)$as being at the origin of the decay chain $\Xi_{c c}^{++} \rightarrow \Sigma_{c}^{++}\left(\rightarrow \Lambda_{c}^{+} \pi^{+}\right)+\bar{K}^{* 0}\left(\rightarrow K^{-} \pi^{+}\right)$. The nonleptonic decay $\Xi_{c c}^{++} \rightarrow \Sigma_{c}^{++}+\bar{K}^{* 0}$ belongs to a class of decays where the quark flavor composition is such that the decay proceeds solely via the factorizing contribution precluding a contamination from internal $W$-exchange. We use the covariant confined quark model previously developed by us to calculate the four helicity amplitudes that describe the dynamics of the transition $\Xi_{c c}^{++} \rightarrow \Sigma_{c}^{++}$induced by the effective $(c \rightarrow u)$ current. We then proceed to calculate the rate of the decay as well as the polarization of the $\Sigma_{c}^{++}$ and $\Lambda_{c}^{+}$baryons and the longitudinal/transverse composition of the $\bar{K}^{* 0}$. We estimate the decay $\Xi_{c c}^{++} \rightarrow \Sigma_{c}^{++} \bar{K}^{* 0}$ to have a branching rate of $B\left(\Xi_{c c}^{++} \rightarrow \Sigma_{c}^{++} \bar{K}^{* 0}\right) \sim 10.5 \%$. As a byproduct of our investigation we have also analyzed the decay $\Xi_{c c}^{++} \rightarrow \Sigma_{c}^{++} \bar{K}^{0}$ for which we find a branching ratio of $B\left(\Xi_{c c}^{++} \rightarrow \Sigma_{c}^{++} \bar{K}^{0}\right) \sim 2.5 \%$.
\end{abstract}

PACS numbers: 12.39.Ki,13.30.Eg,14.20.Jn,14.20.Mr

Keywords: relativistic quark model, light mesons, light and heavy baryons, decay rates and asymmetries

\section{INTRODUCTION}

Very recently the LHCb Collaboration has reported on the discovery of the double charm state $\Xi_{c c}^{++}$[1] found in the invariant mass spectrum of the final state particles $\left(\Lambda_{c}^{+} K^{-} \pi^{+} \pi^{+}\right)$where the $\Lambda_{c}^{+}$baryon was reconstructed in the decay mode $p K^{-} \pi^{+}$. The mass of the new state was given as $3621.40 \pm 0.72 \pm 0.27 \pm 0.14 \mathrm{MeV}$. The central value of the extracted mass is very close to the $3610 \mathrm{MeV}$ value predicted in Ref. [2] in the framework of the one gluon exchange model of de Rujula, Georgi and Glashow [3] which features a Breit-Fermi spin-spin interaction term. It is noteworthy that Ebert et al. predicted a mass of $3620 \mathrm{MeV}$ for the $\Xi_{c c}^{++}$using a relativistic quark-diquark potential model [4]. We interprete the new double charm baryon state found in the $\left(\Lambda_{c}^{+} K^{-} \pi^{+} \pi^{+}\right)$mass distribution as being at the origin of the decay chain $\Xi_{c c}^{++} \rightarrow \Sigma_{c}^{++}\left(\rightarrow \Lambda_{c}^{+} \pi^{+}\right)+\bar{K}^{* 0}\left(\rightarrow K^{-} \pi^{+}\right)$. This decay chain is favored from an experimental point of view since the branching ratios of the daughter particle decays $\Sigma_{c}^{++} \rightarrow \Lambda_{c}^{+} \pi^{+}$and $\bar{K}^{* 0} \rightarrow K^{-} \pi^{+}$are large $(\sim 100 \%$ and, from isospin invariance, $\sim 66 \%$, respectively).

The nonleptonic decay $\Xi_{c c}^{++} \rightarrow \Sigma_{c}^{++}+\bar{K}^{* 0}$ belongs to a class of decays where the quark flavor composition is such that the decay proceeds solely via the factorizing contribution precluding a contamination from internal $W$-exchange. We use the covariant confined quark model previously proposed and developed by us to calculate the four helicity amplitudes that describe the dynamics of the transition $\Xi_{c c}^{++} \rightarrow \Sigma_{c}^{++}$induced by the effective $(c \rightarrow u)$ current. We then proceed to calculate the rate of the decay as well as the polarization of the $\Sigma_{c}^{++}$and $\Lambda_{c}^{+}$baryons and the longitudinal/transverse composition of the $\bar{K}^{* 0}$. The nontrivial helicity composition of the $\bar{K}^{* 0}$ leads to a nontrivial angular decay distribution in terms of the polar angle $\theta_{V}$ formed by the direction of the $K^{-}$in the $\bar{K}^{* 0}$ rest system and the original flight direction of the $\bar{K}^{* 0}$.

Double heavy baryon decays and their magnetic moments were treated by us before in Refs. [5] where we performed a comprehensive study of the semileptonic and radiative decays of double heavy baryons using a covariant quark model. The version of the covariant quark model used in Ref. [5] has recently been improved by incorporating quark confinement in an effective way [6]. For the calculation of the relevant transition $\Xi_{c c}^{++} \rightarrow \Sigma_{c}^{++}$in this paper we use the improved model which we dub the covariant confined quark model (CCQM). The physics of double heavy charm and bottom baryons (mass spectrum and decay properties) has been studied before in a number of papers [7]-[18]. 


\section{MATRIX ELEMENTS, HELICITY AMPLITUDES AND RATE EXPRESSIONS}

The matrix element of the exclusive decay $B_{1}\left(p_{1}, \lambda_{1}\right) \rightarrow B_{2}\left(p_{2}, \lambda_{2}\right)+V\left(q, \lambda_{V}\right)$ is defined by

$$
M\left(B_{1} \rightarrow B_{2}+V\right)=\frac{G_{F}}{\sqrt{2}} V_{i j} V_{k l}^{*} C_{\text {eff }} f_{V} M_{V}\left\langle B_{2}\left|\bar{q}_{2} O_{\mu} q_{1}\right| B_{1}\right\rangle \epsilon^{\dagger \mu}\left(\lambda_{V}\right) .
$$

In the present case $B_{1}=\Xi_{c c}^{++}, B_{2}=\Sigma_{c}^{++}, V=\bar{K}^{* 0}, V_{i j}=V_{u d}=0.97425, V_{k l}^{*}=V_{c s}^{*}=0.974642$. For the effective current strength we use the large $N_{c}$ limit of the relevant effective current combination $C_{\text {eff }}=C_{2}+1 / N_{c} \cdot C_{1}$ to write $C_{\text {eff }}=-0.565$ [19]. The large $N_{c}$ limit has also been used to successfully describe the nonleptonic decays $\Lambda_{b}^{0} \rightarrow \Lambda+J / \psi$ and $\Lambda_{c}^{+} \rightarrow p \phi$ which belong to same class of neutral vector meson decays as $\Xi_{c c}^{++} \rightarrow \Sigma_{c}^{++}+\bar{K}^{* 0}$ which proceed solely via the factorizing contribution (also called internal $W$-emission) in Refs. [20, 21]. The leptonic decay constant is denoted by $f_{V}$. The Dirac string $O^{\mu}$ reads $O^{\mu}=\gamma^{\mu}\left(1-\gamma^{5}\right)$.

The hadronic matrix element $\left\langle B_{2}\left|\bar{q}_{2} O_{\mu} q_{1}\right| B_{1}\right\rangle$ is expressed in terms of four dimensionless invariant form factors $F_{1,2}^{V / A}\left(q^{2}\right)$, viz.

$$
\begin{aligned}
\left\langle B_{2}\left|\bar{q}_{2} \gamma_{\mu} q_{1}\right| B_{1}\right\rangle & =\bar{u}\left(p_{2}, s_{2}\right)\left[\gamma_{\mu} F_{1}^{V}\left(q^{2}\right)-i \sigma_{\mu \nu} \frac{q_{\nu}}{M_{1}} F_{2}^{V}\left(q^{2}\right)\right] u\left(p_{1}, s_{1}\right) \\
\left\langle B_{2}\left|\bar{q}_{2} \gamma_{\mu} \gamma_{5} q_{1}\right| B_{1}\right\rangle & =\bar{u}\left(p_{2}, s_{2}\right)\left[\gamma_{\mu} F_{1}^{A}\left(q^{2}\right)-i \sigma_{\mu \nu} \frac{q_{\nu}}{M_{1}} F_{2}^{A}\left(q^{2}\right)\right] \gamma_{5} u\left(p_{1}, s_{1}\right)
\end{aligned}
$$

where $\sigma_{\mu \nu}=(i / 2)\left(\gamma_{\mu} \gamma_{\nu}-\gamma_{\nu} \gamma_{\mu}\right)$ and all $\gamma$-matrices are defined as in Bjorken-Drell. Here we $\operatorname{drop} F_{3}^{V / A}\left(q^{2}\right)$ form factors, which do not contribute to the decay $B_{1} \rightarrow B_{2}+V$ due to the vector current conservation.

Next we express the vector and axial vector helicity amplitudes $H_{\lambda_{2} \lambda_{V}}^{V / A}$ contributing to the decay $\Xi_{c c}^{++} \rightarrow \Sigma_{c}^{++}+\bar{K}^{\star 0}$ in terms of the invariant form factors $F_{1,2}^{V / A}$, where $\lambda_{V}= \pm 1,0$ and $\lambda_{2}= \pm 1 / 2$ are the helicity components of the vector meson and the baryon $B_{2}$, respectively.

We need to calculate the expression

$$
H_{\lambda_{2} \lambda_{V}}=\left\langle B_{2}\left(p_{2}, \lambda_{2}\right)\left|\bar{q}_{2} O_{\mu} q_{1}\right| B_{1}\left(p_{1}, \lambda_{1}\right)\right\rangle \epsilon^{\dagger \mu}\left(\lambda_{V}\right)=H_{\lambda_{2} \lambda_{V}}^{V}-H_{\lambda_{2} \lambda_{V}}^{A},
$$

where we split the helicity amplitudes into their vector and axial parts. We shall work in the rest frame of the baryon $B_{1}$ with the baryon $B_{2}$ moving in the positive $z$-direction: $p_{1}=\left(M_{1}, \overrightarrow{\mathbf{0}}\right), p_{2}=\left(E_{2}, 0,0,\left|\mathbf{p}_{2}\right|\right)$ and $q=\left(q_{0}, 0,0,-\left|\mathbf{p}_{2}\right|\right)$. The helicities of the $B_{1}, B_{2}$, and $V$ are related by $\lambda_{1}=\lambda_{2}-\lambda_{V}$ leading to the angular momentum restriction $\left|\lambda_{2}-\lambda_{V}\right| \leq 1 / 2$. One has

$$
\begin{array}{rlrl}
H_{\frac{1}{2} 0}^{V}=\sqrt{Q_{-} / M_{V}^{2}}\left(F_{1}^{V} M_{+}+F_{2}^{V} \frac{M_{V}^{2}}{M_{1}}\right), & H_{\frac{1}{2} 0}^{A}=\sqrt{Q_{+} / M_{V}^{2}}\left(F_{1}^{A} M_{-}-F_{2}^{A} \frac{M_{V}^{2}}{M_{1}}\right), \\
H_{\frac{1}{2} 1}^{V}= & \sqrt{2 Q_{-}}\left(-F_{1}^{V}-F_{2}^{V} \frac{M_{+}}{M_{1}}\right), & H_{\frac{1}{2} 1}^{A}=\sqrt{2 Q_{+}}\left(-F_{1}^{A}+F_{2}^{A} \frac{M_{-}}{M_{1}}\right) .
\end{array}
$$

The remaining helicity amplitudes are given by the parity relations $H_{-\lambda_{2},-\lambda_{V}}^{V}=+H_{\lambda_{2}, \lambda_{V}}^{V}$, and $H_{-\lambda_{2},-\lambda_{V}}^{A}=-H_{\lambda_{2}, \lambda_{V}}^{A}$. We use the abbreviations $M_{ \pm}=M_{1} \pm M_{2}, Q_{ \pm}=M_{ \pm}^{2}-M_{V}^{2},\left|\mathbf{p}_{\mathbf{2}}\right|=\lambda^{1 / 2}\left(M_{1}^{2}, M_{2}^{2}, M_{V}^{2}\right) /\left(2 M_{1}\right)$.

The decay width is given by

$$
\Gamma\left(B_{1} \rightarrow B_{2}+V\right)=\frac{G_{F}^{2}}{32 \pi} \frac{\left|\mathbf{p}_{\mathbf{2}}\right|}{M_{1}^{2}}\left|V_{i j} V_{k l}^{*}\right|^{2} C_{\mathrm{eff}}^{2} f_{V}^{2} M_{V}^{2} \cdot\left(\mathcal{H}_{U}+\mathcal{H}_{L}\right)
$$

where we introduce the following combinations of helicity amplitudes

$$
\begin{aligned}
& \mathcal{H}_{U}=\left|H_{\frac{1}{2} 1}\right|^{2}+\left|H_{-\frac{1}{2}-1}\right|^{2} \quad \text { transverse unpolarized } \\
& \mathcal{H}_{L}=\left|H_{\frac{1}{2} 0}\right|^{2}+\left|H_{-\frac{1}{2} 0}\right|^{2} \quad \text { longitudinal unpolarized } .
\end{aligned}
$$

After having set up the spin-kinematical framework of the problem we now turn to the dynamics of the decay process $\Xi_{c c}^{++} \rightarrow \Sigma_{c}^{++} \bar{K}^{* 0}$ which necessarily is model dependent. As remarked on before the sole contribution to the nonleptonic decay $\Xi_{c c}^{++} \rightarrow \Sigma_{c}^{++} \bar{K}^{* 0}$ is the factorizing (or tree graph) contribution. We use the CCQM to calculate the transition matrix element $\Xi_{c c}^{++} \rightarrow \Sigma_{c}^{++}$. 
An important ingredient of the calculation is the choice of the nonlocal interpolating current which we now specify together with the Lagrangian that describes the coupling of the constituent quarks with the double heavy baryon. One has [5, 20]

$$
\begin{array}{rlrl}
\Xi_{c c}^{++}: & \mathcal{L}_{\mathrm{int}}^{\Xi_{c c}^{++}}(x) & =g_{\Xi_{c c}^{++}} \bar{\Xi}_{c c}^{++}(x) \cdot J_{\Xi_{c c}^{++}}(x)+\text { H.c. }, \\
J_{\Xi_{c c}^{++}}(x) & =\int d x_{1} \int d x_{2} \int d x_{3} F_{\Xi_{c c}^{++}}\left(x ; x_{1}, x_{2}, x_{3}\right) \epsilon^{a_{1} a_{2} a_{3}} \gamma^{\mu} \gamma^{5} u^{a_{1}}\left(x_{1}\right) c^{a_{2}}\left(x_{2}\right) C \gamma_{\mu} c^{a_{3}}\left(x_{3}\right), \\
\Sigma_{c}^{++}: & \mathcal{L}_{\mathrm{int}}^{\Sigma_{c}^{++}}(x)=g_{\Sigma_{c}^{++}} \bar{\Sigma}_{c}^{++}(x) \cdot J_{\Sigma_{c}^{++}}(x)+\text { H.c. }, \\
& J_{\Sigma_{c}^{++}}(x)=\int d x_{1} \int d x_{2} \int d x_{3} F_{\Sigma_{c}^{++}}\left(x ; x_{1}, x_{2}, x_{3}\right) \epsilon^{a_{1} a_{2} a_{3}} \gamma^{\mu} \gamma^{5} c^{a_{1}}\left(x_{1}\right) u^{a_{2}}\left(x_{2}\right) C \gamma_{\mu} u^{a_{3}}\left(x_{3}\right) .
\end{array}
$$

Differing from the calculations in [10, 17], which use a quark-diquark picture, we treat each of the three constituent quarks as separate dynamic entities. The propagators $S_{q}(k)=1 /\left(m_{q}-\not k\right)$ for up and charm quarks are taken in a form of free fermion propagators where $m_{q}=m_{u}, m_{c}$ are constituent quark masses fixed in previous analysis of a multitude of hadronic processes in our approach (see, e.g., Refs. [21, 22]): $m_{u}=0.2413 \mathrm{GeV}, m_{c}=1.6722 \mathrm{GeV}$. The compositeness condition of Salam and Weinberg [23] gives one constraint equation between the coupling factors $g_{\Xi_{c c}^{++}}, g_{\Sigma_{c}^{++}}$ and the size parameters $\Lambda_{\Xi_{c c}^{++}}, \Lambda_{\Sigma_{c}^{++}}$charactering the nonlocal distribution $F_{\Xi_{c c}^{++}}\left(x ; x_{1}, x_{2}, x_{3}\right), F_{\Sigma_{c}^{++}}\left(x ; x_{1}, x_{2}, x_{3}\right)$, respectively. As size parameter we use $\Lambda_{\Sigma_{c}^{++}}=0.867 \mathrm{GeV}$ (unified size parameter for the $J^{P}=\frac{1}{2}^{+}$single charm baryons fixed in Refs. [21]) and consider $\Lambda_{\Xi_{c c}^{+}+}$as a free parameter. The size parameters of light and heavy baryons in our approach are varied in the region $0.5-1 \mathrm{GeV}$. Therefore, in our calculations we will vary $\Lambda_{\Xi_{c c}^{++}}$in the interval 0.5-1 GeV. We found that the results for the decay widths $\Gamma\left(\Xi_{c c}^{++} \rightarrow \Sigma_{c}^{++}+\bar{K}^{* 0}\left(\bar{K}^{0}\right)\right)$ are very stable in this region of the size parameter $\Lambda_{\Xi_{c c}^{++}}$. In the following discussion we will indicate the dependence of our results on the choice of $\Lambda_{\Xi_{c c}^{+}}$in the interval $0.5-1 \mathrm{GeV}$ in the form $A \pm \Delta A$.

The leptonic decay constants $f_{K^{*}}=212 \mathrm{MeV}$ and $f_{K}=161.3 \mathrm{MeV}$ evaluated in our approach are in good agreement with data: $f_{K^{*}}=(217 \pm 7) \mathrm{MeV}$ and $f_{K}=(156.1 \pm 0.8) \mathrm{MeV}$ [24].

Let us now list our numerical results for the four helicity amplitudes. They are

$$
\begin{array}{ll}
H_{\frac{1}{2} 0}=\quad 3.0 \pm 0.1 \mathrm{GeV}, & H_{-\frac{1}{2} 0}=-(9.6 \pm 0.4) \mathrm{GeV}, \\
H_{\frac{1}{2} 1}=-(3.0 \pm 0.3) \mathrm{GeV}, & H_{-\frac{1}{2}-1}=10.0 \pm 0.5 \mathrm{GeV} .
\end{array}
$$

For the sum of the moduli squared of the helicity amplitudes one obtains

$$
\begin{aligned}
\mathcal{H}_{N} & =\mathcal{H}_{U}+\mathcal{H}_{L}=210.2 \pm 17.9 \mathrm{GeV}^{2}, \\
\mathcal{H}_{U} & =109.0 \pm 10.2 \mathrm{GeV}^{2}, \\
\mathcal{H}_{L} & =101.2 \pm 7.7 \mathrm{GeV}^{2},
\end{aligned}
$$

which leads to the partial decay width

$$
\Gamma\left(\Xi_{c c}^{++} \rightarrow \Sigma_{c}^{++}+\bar{K}^{* 0}\right)=(0.21 \pm 0.02) \times 10^{12} \mathrm{~s}^{-1} .
$$

We have also analyzed the decay $\Xi_{c c}^{++} \rightarrow \Sigma_{c}^{++}+\bar{K}^{0}$ using the same dynamics as for the decay $\Xi_{c c}^{++} \rightarrow \Sigma_{c}^{++}+\bar{K}^{* 0}$. We obtain

$$
\Gamma\left(\Xi_{c c}^{++} \rightarrow \Sigma_{c}^{++}+\bar{K}^{0}\right)=(0.05 \pm 0.01) \times 10^{12} \mathrm{~s}^{-1}
$$

The $\bar{K}^{* 0}$ mode is about four times stronger than the one including the $\bar{K}^{0}$. In order to convert the partial rate into a branching ratio one would need the total width or, equivalently, the lifetime value of the $\Xi_{c c}^{++}$. Neither of these are known experimentally. There have been several attempts to calculate the lifetime of the $\Xi_{c c}^{++}$based on the optical theorem for the inclusive decay width combined with the Operator Product Expansion for the transition currents together with a heavy quark mass expansion. The results are in the range of $430 \mathrm{fs}-670 \mathrm{fs}[8,13$ ]. As a median value we take $\tau_{\Xi_{c c}^{++}}=500 \mathrm{fs}$. For the branching ratios we obtain

$$
\begin{aligned}
B\left(\Xi_{c c}^{++} \rightarrow \Sigma_{c}^{++}+\bar{K}^{* 0}\right) & =\left(\frac{\tau_{\Xi_{c c}^{++}}}{500 \mathrm{fs}}\right) \cdot(10.5 \pm 1) \% \\
B\left(\Xi_{c c}^{++} \rightarrow \Sigma_{c}^{++}+\bar{K}^{0}\right) & =\left(\frac{\tau_{\Xi_{c c}^{++}}}{500 \mathrm{fs}}\right) \cdot(2.5 \pm 0.5) \%
\end{aligned}
$$




\section{POLARIZATION, LONGITUDINAL/TRANSVERSE HELICITY FRACTION AND ANGULAR DECAY DISTRIBUTIONS}

We treat the decaying $\Xi_{c c}^{++}$as being unpolarized. In principle, the $\Xi_{c c}^{++}$could acquire a nonzero transverse polarization in the hadronic production process. However, since one is averaging over the rapidities of the production process the $\Xi_{c c}^{++}$is effectively unpolarized (for more details see [22]). The baryon-side decay $\Sigma_{c}^{++} \rightarrow \Lambda_{c}^{+} \pi^{+}$is a strong decay and, even though the $\Sigma_{c}^{++}$is polarized, the decay $\Sigma_{c}^{++} \rightarrow \Lambda_{c}^{+} \pi^{+}$possesses zero analyzing power to resolve the polarization of the $\Sigma_{c}^{++}$, i.e. the azimuthal angle and the helicity angle decay distribution of the decay $\Sigma_{c}^{++} \rightarrow \Lambda_{c}^{+} \pi^{+}$ is uniform. For the meson-side decay $\bar{K}^{* 0} \rightarrow K^{-} \pi^{+}$one obtains the angular decay distribution

$$
\begin{aligned}
\frac{d \Gamma\left(\Xi_{c c}^{++} \rightarrow \Sigma_{c}^{++}+\bar{K}^{* 0}\left(\rightarrow K^{-} \pi^{+}\right)\right)}{d \cos \theta_{V}} & =B\left(\bar{K}^{* 0} \rightarrow K^{-} \pi^{+}\right) \frac{G_{F}^{2}}{32 \pi} \frac{\left|\mathbf{p}_{\mathbf{2}}\right|}{M_{1}^{2}}\left|V_{i j} V_{k l}^{*}\right|^{2} C_{\mathrm{eff}}^{2} f_{V}^{2} M_{V}^{2} \mathcal{H}_{N} \\
& \times\left(\frac{3}{2} \cos ^{2} \theta_{V} \mathcal{F}_{L}+\frac{3}{4} \sin ^{2} \theta_{V} \mathcal{F}_{T}\right)
\end{aligned}
$$

where $B\left(\bar{K}^{* 0} \rightarrow K^{-} \pi^{+}\right)=2 / 3$ is the branching ratio of the decay $\bar{K}^{* 0} \rightarrow K^{-} \pi^{+}$. The angular decay distribution (13) involves the helicity fractions of the $\bar{K}^{* 0}$ defined by

$$
\mathcal{F}_{L}=\frac{\left|H_{\frac{1}{2} 0}\right|^{2}+\left|H_{-\frac{1}{2} 0}\right|^{2}}{\mathcal{H}_{N}}=0.48 \pm 0.01, \quad \mathcal{F}_{T}=\frac{\left|H_{\frac{1}{2} 1}\right|^{2}+\left|H_{-\frac{1}{2}-1}\right|^{2}}{\mathcal{H}_{N}}=0.52 \pm 0.01 .
$$

This has to be compared to the unpolarized case $\mathcal{F}_{L}=1 / 3$ and $\mathcal{F}_{T}=2 / 3$ which is e.g. realized at the zero recoil point $q^{2}=\left(M_{1}-M_{2}\right)^{2}$ where there is only the axial vector $S$-wave excitation of the final $\left(\Sigma_{c}^{++} \bar{K}^{* 0}\right)$-state with $\sqrt{2} H_{1 / 20}^{A}=H_{1 / 21}^{A}$ ("allowed Fermi-Teller transition"). Our results for the helicity fractions considerably deviate from their unpolarized values leading to a pronounced $\cos \theta_{V}$-dependence of the angular decay distribution (13) which is quite close to $W\left(\theta_{V}\right) \sim 3 / 8\left(1+\cos ^{2} \theta_{V}\right)$.

The longitudinal polarization of the daughter baryon $\Sigma_{c}^{++}$depends on the polar emission angle $\theta_{V}$ via

$$
P_{\Sigma_{c}^{++}}\left(\cos \theta_{V}\right)=\frac{\frac{3}{4} \sin ^{2} \theta_{V}\left(\left|H_{\frac{1}{2} 1}\right|^{2}-\left|H_{-\frac{1}{2}-1}\right|^{2}\right)+\frac{3}{2} \cos ^{2} \theta_{V}\left(\left|H_{\frac{1}{2} 0}\right|^{2}-\left|H_{-\frac{1}{2} 0}\right|^{2}\right)}{\frac{3}{4} \sin ^{2} \theta_{V}\left(\left|H_{\frac{1}{2} 1}\right|^{2}+\left|H_{-\frac{1}{2}-1}\right|^{2}\right)+\frac{3}{2} \cos ^{2} \theta_{V}\left(\left|H_{\frac{1}{2} 0}\right|^{2}+\left|H_{-\frac{1}{2} 0}\right|^{2}\right)} .
$$

When averaged over $\cos \theta_{V}$ (one has to integrate the numerator and denominator separately) one has

$$
P_{\Sigma_{c}^{++}}=\frac{\left(\left|H_{\frac{1}{2} 1}\right|^{2}-\left|H_{-\frac{1}{2}-1}\right|^{2}\right)+\left(\left|H_{\frac{1}{2} 0}\right|^{2}-\left|H_{-\frac{1}{2} 0}\right|^{2}\right)}{\mathcal{H}_{N}}=-(0.83 \pm 0.01) .
$$

As mentioned before the polarization of the $\Sigma_{c}^{++}$is not measurable in its strong decays. However, the $\Sigma_{c}^{++}$transfers its polarization to the $\Lambda_{c}^{+}$in the strong decay $\Sigma_{c}^{++} \rightarrow \Lambda_{c}^{+} \pi^{+}$. The average longitudinal polarization of the $\Lambda_{c}^{+}$can be calculated to be (we average over $\cos \theta_{V}$ ):

$$
P_{\Lambda_{c}^{+}}\left(\theta_{B}\right)=\frac{\left|H_{\frac{1}{2} 0}\right|^{2}-\left|H_{-\frac{1}{2} 0}\right|^{2}+\left|H_{\frac{1}{2} 1}\right|^{2}-\left|H_{-\frac{1}{2}-1}\right|^{2}}{\mathcal{H}_{N}} \cos \theta_{B}=-(0.83 \pm 0.01) \cos \theta_{B}
$$

where $\theta_{B}$ is the angle between the direction of the $\Lambda_{c}^{+}$and the original flight direction of the $\Sigma_{c}^{++}$, all in the rest frame of the $\Sigma_{c}^{++}$.

For the decay $\Xi_{c c}^{++} \rightarrow \Sigma_{c}^{++}+\bar{K}^{0}$ we find a slightly larger value of the longitudinal polarization of the $\Sigma_{c}^{++}$given by

$$
P_{\Sigma_{c}^{++}}\left(\Xi_{c c}^{++} \rightarrow \Sigma_{c}^{++}+\bar{K}^{0}\right)=\frac{\left|H_{\frac{1}{2} t}\right|^{2}-\left|H_{-\frac{1}{2} t}\right|^{2}}{\mathcal{H}_{S}}=-(0.95 \pm 0.02) .
$$

In principle, the polarization of the $\Lambda_{c}^{+}$can be analyzed in its weak decay $\Lambda_{c}^{+} \rightarrow p K^{-} \pi^{+}$. For example, one could attempt to measure nonvanishing values of the expectation value $\left\langle\cos \theta_{i}\right\rangle$ where $\theta_{i}$ is the polar angle between the polarization direction of the $\Lambda_{c}^{+}$and either one of the three decay particles $\left(i=p, K^{+}, \pi^{-}\right)$or the normal of the decay plain (see an exemplary analysis of a weak $(1 \rightarrow 3)$-particle decay in e.g. [25]). To our knowledge the weak decay $\Lambda_{c}^{+} \rightarrow p K^{-} \pi^{+}$has not been completely calculated yet except for an analysis of the subchannels $\Lambda_{c}^{+} \rightarrow p \bar{K}^{* 0}$ and $\Lambda_{c}^{+} \rightarrow \Delta^{++} K^{-}$[26]. 


\section{SUMMARY AND CONCLUSION}

We have discussed in some detail the possibility that the new double charm state found in the invariant mass distribution of $\left(\Lambda_{c}^{+} K^{-} \pi^{+} \pi^{+}\right)$can be attributed to the decay chain $\Xi_{c c}^{++} \rightarrow \Sigma_{c}^{++}\left(\rightarrow \Lambda_{c}^{+} \pi^{+}\right)+\bar{K}^{* 0}\left(\rightarrow K^{-} \pi^{+}\right)$. The hypothesis can be tested experimentally by looking at the decay distributions of the particles involved in the cascade decay. For once one can check whether there are significant peaks at the $\Sigma_{c}^{++}$and $\bar{K}^{* 0}$ masses in the $\left(\Lambda_{c}^{+} \pi^{+}\right)$and $\left(K^{-} \pi^{+}\right)$invariant mass distributions, respectively. If there is a significant continuum background one would have to place relevant cuts on the invariant mass distribution to obtain the appropriate cascade decay channels discussed in this paper. One can then go on and check on the angular decay distributions in the respective cascade decays which have been written down in this paper. We have also discussed the decay $\Xi_{c c}^{++} \rightarrow \Sigma_{c}^{++}+\bar{K}^{0}$ which we predict to have a branching ratio four times smaller than that of the decay $\Xi_{c c}^{++} \rightarrow \Sigma_{c}^{++}+\bar{K}^{* 0}$. It would nevertheless be interesting to experimentally search for this decay mode.

It would also be worthwhile to experimentally check on further nonleptonic decay channels of the double charm state $\Xi_{c c}^{++}$(see also Refs. [16]). For once there are the decay channels $\Xi_{c c}^{++} \rightarrow \Xi_{c}^{*+}\left(\rightarrow \Xi_{c}^{0+\pi^{+}}\right)+\pi^{+}\left(\rho^{+}\right)$and $\Xi_{c c}^{++} \rightarrow \Xi_{c}^{*++}(\rightarrow$ $\left.\Xi_{c}^{+}+\pi^{+}\right)+\rho^{0}$. Experimentally more challenging would be the decay channels $\Xi_{c c}^{++} \rightarrow \Xi_{c}^{\prime}\left(\rightarrow \Xi_{c}^{+}+\gamma\right)+\pi^{+}\left(\rho^{+}\right)$, and $\Xi_{c c}^{++} \rightarrow \Xi_{c}^{*++}\left(\rightarrow \Xi_{c}^{+}+\pi^{+}\right)+\pi^{0}$ because their detection would require photon identification. The above two-body nonleptonic decay modes belong to the same class of processes as the decays $\Xi_{c c}^{++} \rightarrow \Sigma_{c}^{++}+\bar{K}^{* 0}\left(\bar{K}^{0}\right)$ in that they are solely contributed to by the factorizing (or tree graph) contribution. A possible $W$-exchange contribution (color commensurate " $\mathrm{C}$ " in the terminology of [27]) is forbidden by the Körner, Pati, Woo theorem [28]. The calculation of the above rates proceeds in the same way as the calculation in this paper and will be the subject of a future publication [29].

\section{Acknowledgments}

This work was funded by the German Bundesministerium für Bildung und Forschung (BMBF) under Project 05P2015 - ALICE at High Rate (BMBF-FSP 202): "Jet- and fragmentation processes at ALICE and the parton structure of nuclei and structure of heavy hadrons", by CONICYT (Chile) PIA/Basal FB0821, by Tomsk State University Competitiveness Improvement Program and the Russian Federation program "Nauka" (Contract No. 0.1764.GZB.2017). The research is carried out at Tomsk Polytechnic University within the framework of Tomsk Polytechnic University Competitiveness Enhancement Program grant. M.A.I. acknowledges the support from PRISMA Cluster of Excellence (Mainz Uni.). M.A.I. and J.G.K. thank the Heisenberg-Landau Grant for partial support.

[1] R. Aaij et al. [LHCb Collaboration], arXiv:1707.01621 [hep-ex].

[2] J. G. Körner, M. Krämer and D. Pirjol, Prog. Part. Nucl. Phys. 33, 787 (1994) hep-ph/9406359.

[3] A. De Rujula, H. Georgi and S. L. Glashow, Phys. Rev. D 12, 3589 (1975).

[4] D. Ebert, R. N. Faustov, V. O. Galkin and A. P. Martynenko, Phys. Rev. D 66, 014008 (2002) hep-ph/0201217.

[5] A. Faessler, T. Gutsche, M. A. Ivanov, J. G. Körner and V. E. Lyubovitskij, Phys. Lett. B 518, 55 (2001) hep-ph/0107205; A. Faessler, T. Gutsche, M. A. Ivanov, J. G. Korner, V. E. Lyubovitskij, D. Nicmorus and K. Pumsa-ard, Phys. Rev. D 73, 094013 (2006) hep-ph/0602193; Phys. Rev. D 80, 034025 (2009) arXiv:0907.0563 [hep-ph]]; AIP Conf. Proc. 1257, 311 (2010); T. Branz, A. Faessler, T. Gutsche, M. A. Ivanov, J. G. Körner, V. E. Lyubovitskij and B. Oexl, Phys. Rev. D 81, 114036 (2010) arXiv:1005.1850 [hep-ph]].

[6] T. Branz, A. Faessler, T. Gutsche, M. A. Ivanov, J. G. Körner and V. E. Lyubovitskij, Phys. Rev. D 81, 034010 (2010) arXiv:0912.3710 [hep-ph]].

[7] S. Fleck and J. M. Richard, Prog. Theor. Phys. 82, 760 (1989).

[8] V. V. Kiselev, A. K. Likhoded and A. I. Onishchenko, Phys. Rev. D 60, 014007 (1999) hep-ph/9807354; V. V. Kiselev and A. K. Likhoded, Phys. Usp. 45, 455 (2002) [Usp. Fiz. Nauk 172, 497 (2002)] hep-ph/0103169.

[9] S. S. Gershtein, V. V. Kiselev, A. K. Likhoded and A. I. Onishchenko, Phys. Rev. D 62, 054021 (2000); V. V. Kiselev, A. K. Likhoded and A. I. Onishchenko, Eur. Phys. J. C 16, 461 (2000) hep-ph/9901224; V. V. Kiselev, A. K. Likhoded, O. N. Pakhomova and V. A. Saleev, Phys. Rev. D 66, 034030 (2002) hep-ph/0206140; A. V. Kiselev, A. V. Berezhnoy and A. K. Likhoded, arXiv:1706.09181 [hep-ph].

[10] D. Ebert, R. N. Faustov, V. O. Galkin and A. P. Martynenko, Phys. Rev. D 70, 014018 (2004) hep-ph/0404280; Phys. Rev. D 77, 079903(E) (2008).

[11] C. Albertus, E. Hernandez, J. Nieves and J. M. Verde-Velasco, Eur. Phys. J. A 32, 183 (2007) hep-ph/0610030; Eur. Phys. J. A 36, 119(E) (2008); E. Hernandez, J. Nieves and J. M. Verde-Velasco, Phys. Lett. B 663, 234 (2008) arXiv:0710.1186 [hep-ph]]; C. Albertus, E. Hernandez and J. Nieves, Phys. Lett. B 683, 21 (2010) arXiv:0911.0889 [hep-ph]]; C. Albertus, 
E. Hernndez and J. Nieves, Phys. Lett. B 704, 499 (2011) arXiv:1108.1296 [hep-ph]]; C. Albertus, E. Hernandez and J. Nieves, Phys. Rev. D 85, 094035 (2012) arXiv:1202.4861 [hep-ph]].

[12] J. M. Flynn and J. Nieves, Phys. Rev. D 76, 017502 (2007) arXiv:0706.2805 [hep-ph]]; Phys. Rev. D 77, 099901(E) (2008).

[13] C. H. Chang, T. Li, X. Q. Li and Y. M. Wang, Commun. Theor. Phys. 49, 993 (2008) arXiv:0704.0016 [hep-ph]].

[14] W. Roberts and M. Pervin, Int. J. Mod. Phys. A 24, 2401 (2009) arXiv:0803.3350 [nucl-th]].

[15] M. Karliner and J. L. Rosner, Phys. Rev. D 90, 094007 (2014) arXiv:1408.5877 [hep-ph]; arXiv:1706.06961 [hep-ph]; arXiv:1707.07666 [hep-ph].

[16] R. H. Li, C. D. Lü, W. Wang, F. S. Yu and Z. T. Zou, Phys. Lett. B 767, 232 (2017) arXiv:1701.03284 [hep-ph]]; F. S. Yu, H. Y. Jiang, R. H. Li, C. D. Lü, W. Wang and Z. X. Zhao, arXiv:1703.09086 [hep-ph]. W. Wang, Z. P. Xing and J. Xu, arXiv:1707.06570 [hep-ph].

[17] W. Wang, F. S. Yu and Z. X. Zhao, arXiv:1707.02834 [hep-ph].

[18] H. X. Chen, Q. Mao, W. Chen, X. Liu and S. L. Zhu, arXiv:1707.01779 [hep-ph]; H. S. Li, L. Meng, Z. W. Liu and S. L. Zhu, arXiv:1707.02765 [hep-ph]; B. O. Kerbikov, arXiv:1707.04031 [hep-ph].

[19] G. Buchalla, A. J. Buras and M. E. Lautenbacher, Rev. Mod. Phys. 68, 1125 (1996) hep-ph/9512380.

[20] M. A. Ivanov, V. E. Lyubovitskij, J. G. Körner and P. Kroll, Phys. Rev. D 56, 348 (1997) hep-ph/9612463; M. A. Ivanov, J. G. Körner, V. E. Lyubovitskij and A. G. Rusetsky, Phys. Rev. D 57, 5632 (1998) hep-ph/9709372; Mod. Phys. Lett. A 13, 181 (1998) hep-ph/9709325.

[21] T. Gutsche, M. A. Ivanov, J. G. Körner, V. E. Lyubovitskij and P. Santorelli, Phys. Rev. D 88, 114018 (2013) arXiv:1309.7879 [hep-ph]]; Phys. Rev. D 92, 114008 (2015) arXiv:1510.02266] [hep-ph]].

[22] T. Gutsche, M. A. Ivanov, J. G. Körner, V. E. Lyubovitskij, V. V. Lyubushkin and P. Santorelli, Phys. Rev. D 96, 013003 (2017) arXiv:1705.07299 [hep-ph]].

[23] A. Salam, Nuovo Cim. 25, 224 (1962); S. Weinberg, Phys. Rev. 130, 776 (1963); K. Hayashi, M. Hirayama, T. Muta, N. Seto and T. Shirafuji, Fortsch. Phys. 15, 625 (1967); G. V. Efimov and M. A. Ivanov, The Quark Confinement Model of Hadrons, (IOP Publishing, Bristol \& Philadelphia, 1993).

[24] C. Patrignani et al. (Particle Data Group), Chin. Phys. C 40, 100001 (2016).

[25] J. G. Körner and D. Pirjol, Phys. Rev. D 60, 014021 (1999) hep-ph/9810511.

[26] B. König, J. G. Körner and M. Krämer, Phys. Rev. D 49, 2363 (1994) hep-ph/9310263.

[27] A. K. Leibovich, Z. Ligeti, I. W. Stewart and M. B. Wise, Phys. Lett. B 586, 337 (2004) hep-ph/0312319.

[28] J. G. Körner, Nucl. Phys. B 25282 (1971); J. C. Pati and C. H. Woo, Phys. Rev. D 3, 2920 (1971).

[29] T. Gutsche, M. A. Ivanov, J. G. Körner and V. E. Lyubovitskij, to be published. 